\title{
Influence of Stretching-Segment Storage Length on Urban Traffic Flow in Signalized Intersection
}

\author{
Weiwei Guo, Wuhong Wang* \\ School of Mechanical Engineering, Beijing Institute of Technology \\ Beijing 100081, China \\ Geert Wets \\ Transportation Research Institute, Hasselt University, \\ Wetenschapspark 5 bus 6,3590 Diepenbeek, Belgium \\ Dianhai Wang \\ College of Civil Engineering and Architecture, Zhejiang University \\ Hangzhou 310058, China \\ Yan Mao \\ School of Mechanical Engineering, Beijing Institute of Technology \\ Beijing 100081, China \\ Xiaobei Jiang ${ }^{1,2}$ \\ 1Department of Transportation Engineering, Beijing Institute of Technology, Beijing 100081, China \\ 2Lehrstuhl für Ergonomie, Technische Universität München, München 85747, Germany \\ Hongwei Guo \\ School of Mechanical Engineering, Beijing Institute of Technology \\ Beijing 100081, China \\ Received 20 July 2011 \\ Accepted 29 November 2011
}

\begin{abstract}
Intelligent traffic control is influenced by Stretching-segment design, parameters optimization of traffic control have to consider saturation flow rate in approach. Many intersections with different stretching-segment design forms are selected to study on saturation flow rate at peak rush hour and ordinary time hour, the characteristic of saturation flow rate in green time is analyzed. Based on the real traffic situation, the influence of the length of stretching-segment storage to straight-cross flow rate was explored in this study. The data showed that the duration and stability of saturation flow rate were highly influenced by the length of stretching-segment storage. A probabilistic computing model was proposed, which analyzed the effect of the length of stretching-segment to the flow rate and described the impact of vehicles in the upstream. Finally, the attenuate regulation of the straight-cross flow rate in green phase is developed by the analysis of vehivle's blocking.

Keywords: Stretching-segment, Intelligent Traffic control, Parameter optimization, Saturation flow rate, Probabilistic computing
\end{abstract}

\footnotetext{
* Corresponding author: wangwuhong@bit.edu.cn
} 


\section{Introduction}

During the channelizing design process of the intersection, Broadening the approach appropriately to increase the number of lanes Intersection to is an effective way to improve traffic capacity. The design of Stretching-Segment can directly affect the traffic flow rate of approach. Experts in China and abroad have launched a long-term research about this. Clayton ( 1941 ) first proposed the motion characteristics of the traffic flow through the intersection stop line during the single lane saturation release process ${ }^{1}$, then experts Wardrop 、 Webster $^{2}$ and Cobbe etc. Continue to use and developed the Clayton model. About the calculation of saturation flow, Since the 1950s till now, has formed a number of empirical formula, including Webster and Cobbe methods, Akcelik method ${ }^{3}$, Kimber method ${ }^{4}$, the cumulative curve method $^{5}$ and HCM method ${ }^{6}$ and so on. These methods togrther show the two features of the saturation flow rate: (1) the maximum flow rate in flow rate; (2) can persist for some time. However, in subsequent theory and practice, scholars have increasingly found that the saturation flow rate is actually unworthy of the title, only appear under the ideal traffic state, This was reflected by the "stability" of the saturation flow rate does not really stable, Affected by a variety of factors' interference, saturation flow rate tend to last a short time after the apparent attenuation, or showing volatility, for this reason, scholars from various countries conducted a series of related research. Michalopoulos (1978) studied the estimates question of Left-turning saturated flow rate in both Signal intersection and no signal intersection ${ }^{7}$. Soegondo (1983) thought that saturation flow is the maximum traffic volume of approacj during the green and yellow light period and limited to the through traffic flow and left-turning traffic flow ${ }^{8}$. Khosla

(2006) studied the running effect of saturated flow rate under a longer green time at an approach Intersection of Texas $^{9}$. Wang (2010) studied the Left-turning saturated flow rate model by considering left-turning operation characteristic and the impact on the traffic flow ${ }^{10}$.

In summary, during the research process of the saturation flow rate, Defining and measuring method of the saturation flow rate should be considered preferentialon preliminary stage, then gradually consider the influence of other factors: Lane width, traffic driving direction, vehicle types, loss of time etc., Saturation flow rate is defined as a maximum flow rate which can persist for some time, besides,some scholars believe that the saturation flow rate is not stable and consistent but has certain volatility, Especially the long green light will lead to a decline in saturation flow rate ${ }^{11,12}$. Although the saturation flow rate is not stable and consistent in practice has been noticed, the research about saturated flow rate steady continuous rule, fluctuation and decline reason is not enough, the study of channelizing design effect on saturation flow rate is more rare, And because the through traffic flow is usually the main traffic flow at approaches, therefore the main research target of the current paper is: the design of Stretching-Segment effect on through traffic flow rate.

\section{2 basic design philosophy of Stretching-Segment}

The broaden of the intercection Import-way consists of Stretching-transition section and Stretching-Segment, Stretching-trandition section is part of transition between the Connecting-road and Stretching-segment. The Stretching-Segment is used to compress and storage vehicles that arrive during the Red-light, the compressed Line-up vehicles during the Green-light was released efficiently ${ }^{13,14}$, which improve the traffic efficiency during the Green-light, then improve the traffic capacity of the intersection.

The design of Import-way of the signal-intersection includes the horizontal-design and vertical-design, the horizontal-design is mainly determine the number and attributes of import-way, the study about the influence of horizontal of Stretching-segment to the flow-rate is more mature; the vertical-design is mainly design the length of import-way ${ }^{15,16}$. How to design the length of Stretching-Segment and Stretching-transition section, it has developed a more sophisticated algorithm at home and abroad, the relevant standards and norms studied by United States ,Japan and China, has the same design ideas basically, just the specific parameters differ according to national traffic characteristics. Nevertheless, due to the limitations of the road space, random fluctuations of traffic flow, characteristics of different drivers, it is still difficult to completely avoid the adverse effects of the shortage of Stretching-Segment storage length to traffic flow rata. 


\section{Data Collection}

Based on the real traffic situation, the traffic survey which contains the flow rates under different stretching-segments needs to be done for this research. Therefore, the data of 4 intersections in Beijing were observed in peak hours, such as the Xizhaosi Street Guangming Road intersection, the Zhongguancun Street Zhichun Road intersection, East Huayuan Road Longxiang Road intersection and the Guanganmen Inner Street - Baiguang Road intersection.

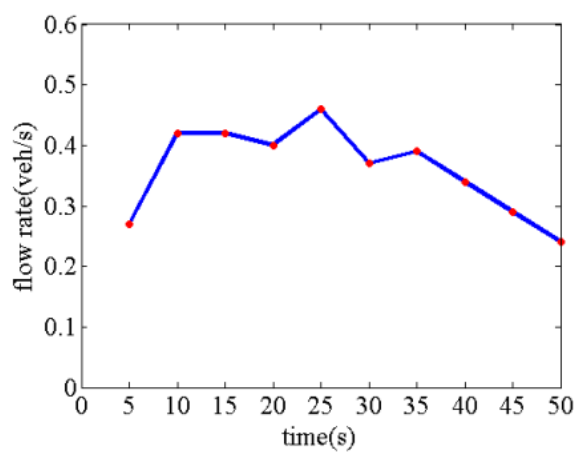

Fig.1 Without Stretching-segment

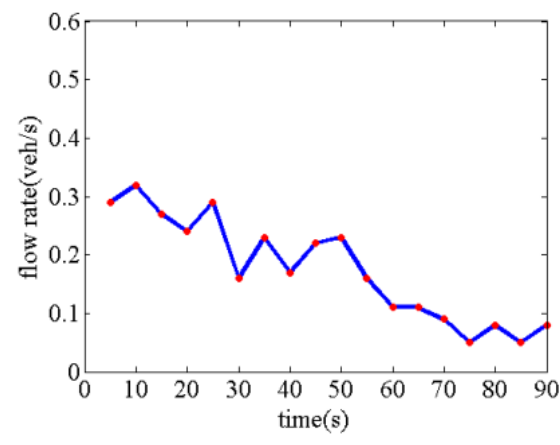

Fig.2 With a 35m Stretching-segment

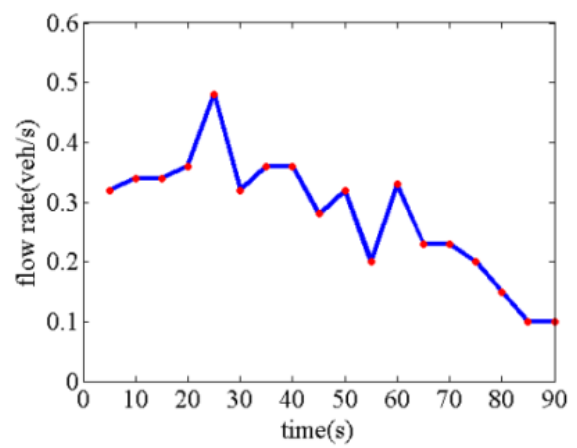

Fig.3 With a 50m Stretching-segment

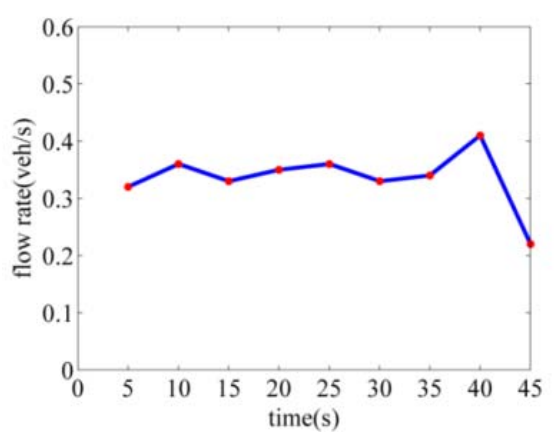

Fig.4 With a 130m Stretching-segment

From those flow rate data under different stretching-segments, it is easy to find that all of the flow rates approached saturation flow rates in 10s and were more stable with the increasing length of the stretching-segments. However, the flow rates became waved in the end of the green phase. The main reason is that vehicles will disturb each other when entering the stretching-segment under the condition that the stretching-segment storage is not enough, the vehicle queue overflows and vehicles complete crossing. That is to say, the efficience of vehicles crossing in the intersection is decreased.

\section{Methodology}

As shown in Fig.5, the straight-cross vehicle is mainly disturbed by th left-turn vehicle. When the left-turn vehicle queue overflows, the straight-cross vehicle will be disturbed ${ }^{17}$. So, the number of vehicles completed straight-crossing task will decrease and traffic accident will even happen in the upstream of the stretching-segment.

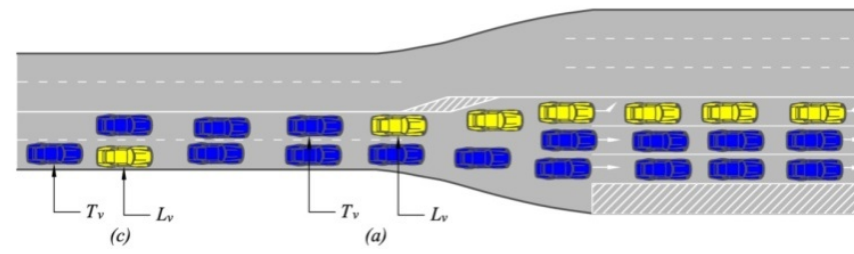

Fig.5 Illustration of the approach

\subsection{Important Assumptions}

In order to analyze the process that straight-cross vehicle is disturbed by the left-turn vehicle and the flow rate of straight-cross vehicles, some imprtant assumptions 
are defined as follows.

(a) The entrance of the intersection is shown in

Figure 2.2.4;

(b) The number of arrival vehicles fits the Poisson Distribution; ignored;

(c) The effection of the right-turn vehicles should be

(d) The straight-cross and left-turn vehicle rates are the same in $L_{a n e}$ and $L_{a n e}$, and they have the same input.

Where,

$n_{v}$ — the number of straight-cross and left-turn vehicles in recorded time, veh;

$n_{v T}$ the number of straight-cross vehicles in recorded time, veh;

$n_{v L}$ — the number of left-turn vehicles in recorded time, veh;

$n_{\text {lane }}$ the number of vehicle lanes in recorded segment;

$n_{\text {laneT }}$ the number of straight-cross vehicle lanes in the stretching-segment;

$n_{\text {lane }}$ - the number of left-turn vehicle lanes in the stretching-segment;

$n_{a v}$ the maximum number of vehicles which is possible in a vehicle lane of the stretching-segment, veh;

$n_{a v T L} \longrightarrow$ the total number of straight-cross and left-turn vehicles which is possible in the stretching-segment, veh;

$n_{a v T}$ the total number of straight-cross vehicels which is possible in the stretching-segment, veh;

$n_{a v L}$ the total number of left-turn vehicels which is possible in the stretching-segment, veh;

$\rho_{T}$ — the straight-cross vehicle rate;

$\rho_{L}$ — the left-turn vehicle rate, $\rho_{L}=1-\rho_{T}$;

$P_{L}(x)$ the probability that $\mathrm{x}$ vehicles enter the intersection;

$P_{J L}$ — the blocking probability of straight-cross vehicles caused by left-turn vehicles.

\subsection{Blocking Probability}

The straight-cross and left-turn vehicels are all fitted with the Binominal Distribution, where the number of vehicles entered the intersection is $n_{v}$, the probability of vehicles which choose straight-crossing is $\rho_{T}$ and the probability of vehicles which choose left-turning is $\rho_{L}$ $\left(\rho_{T}=1-\rho_{L}\right)$.

The regularity of distribution of left-turn vehicles can be formulated by Equation (1).

$$
\begin{gathered}
P_{L}\left(n_{v L}\right)=C_{n_{v}}^{n_{v L}} \rho_{L}^{n_{v L}}\left(1-\rho_{L}\right)^{n_{v}-n_{v L}} \\
\text { for } n_{v L}=0,1,2, \ldots \ldots, n_{v}
\end{gathered}
$$

$n_{a v L}$ is the number of left-turn vehicles which is possible in the stretching-segment and $P_{J L}$ represents the blocking probability of straight-cross vehicles caused by left-turn vehicles. When $n_{v L}>n_{a v L}$, the left-turn vehicles overflow and disturb the straight-cross vehicles, $P_{J L}$ could be formulated by Equation (2).

$$
P_{J L}=\sum_{n_{v L}=n_{a L L}+1}^{n_{v}} P_{L}\left(n_{v L}\right)
$$

The numerical analysis method was used to analyze the performance of Equation (1) and (2). A set of curves can be achieved when $n_{v} \in(1 \sim 40), n_{a v}=5, \quad \rho_{L} \in(0.1 \sim 0.9)$, as see in Figure 6 . The curve from right to left followed by $n_{v} \in(1 \sim 40)$. When $n_{v}$ is less and the stretching-segment is larger than the traffic demand, $P_{J L}$ is slowly increased with the growth of $\rho_{L}$. While $n_{v}$ becomes larger, $P_{J L}$ is highly increased and the inflection point of $P_{J L}=1$ moves forward. When $n_{v}=40$ and $\rho_{L}=0.3, P_{L J}$ is almost equal to 1 . The regularity of distribution of the blocking probability of straight-cross vehicles caused by left-turn vehicles under different number of arrival vehivles is shown in Figure 6, which indicates that the larger the traffic demand than the traffic supply the higher the blocking probability is.

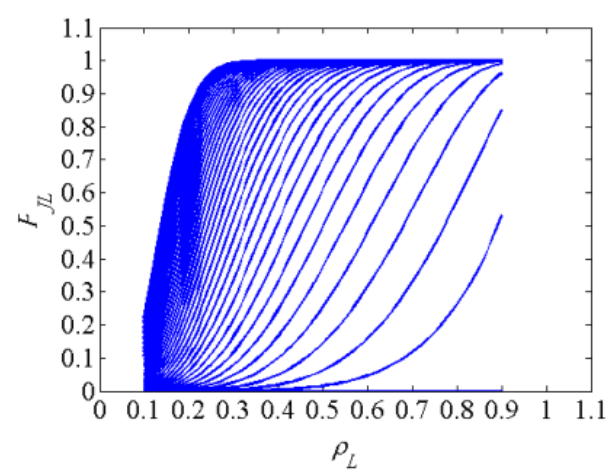

Fig.6 Left-turn vehicle was disturbed by the straight-cross Vehicle, $n_{v} \in(1 \sim 40)$

\subsection{Blocking condition}

The blocking probability distribution of straight-cross vehicles caused by left-turn vehicles was analyzed. However, as shown in Figure 5, when the left-turn vehicles overflow, there is still a lane for 
straight-cross vehicle to cross. So, it is necessary to analyze the expectation of straight-cross vehicles when the left-turn vehicles overflow, because of the different blocking degrees under different flow rate.

The number of left-turn vehicles in Lane $_{1}$ can be calculated by Equation (3).

$$
n_{v L J}=n_{v L}-n_{a v L}
$$

The position $j_{1}$ of the $i_{1}$ th left-turn vehicle in the queue can be defined by Equation (4), where the $O_{Q L J}$ represents the position matrix of the overflowed left-turn vehicles.

$$
\begin{aligned}
& O_{Q L J}\left(i_{1}\right)=j_{1}, i_{1}=1,2, \ldots \ldots, n_{v L J}, \\
& j_{1}=n_{a v}+1, \quad n_{a v}+2, \ldots \ldots, \quad n_{a v}+n_{v L J}+\frac{n_{v T}-n_{a v T}}{2}
\end{aligned}
$$

If the order of the overflowed vehicles are only considered and the $j_{1}=n_{a v}+1$ can be simplified to be $j_{1}=1$, the Equation (4) can be described as the Equation (5).

$$
\begin{aligned}
& O_{Q L J}\left(i_{1}\right)=j_{1}, \\
& i_{1}=1,2, \ldots \ldots, n_{v L J}, \\
& j_{1}=1,2, \ldots \ldots, \quad n_{v L J}+\frac{n_{v T}-n_{a v T}}{2}
\end{aligned}
$$

If the array $O_{A L}$ is the arrival order of the left-turn vehicles and $j_{2}$ is the order of the $i_{2}$ th left-turn vehicle, the Equation (6) can be acquired.

$$
\begin{aligned}
& O_{A L}\left(i_{2}\right)=j_{2}, \\
& i_{2}=1,2, \ldots \ldots, n_{v L S}, \\
& j_{2}=1,2, \ldots \ldots, n_{v}
\end{aligned}
$$

As every overflowed left-turn vehicle can only effect the straight-cross vehicle in the upstream of the vehicle queue, the position of the first overflowed vehicle will determin the total number of the blocked straight-cross vehicles and the affections by other overflowed left-turn vehicles are all contained within the first overflowed vehivle'impact.

The Equation (7) can be obtained when the arrival order of the vehivles in the reserved left-turn vehicle lane fits certain conditions.

$$
O_{A L}\left(i_{2}\right) \leq n_{a v T}+2+i_{2}-1 \text { for } 1 \leq i_{2} \leq n_{a v L}
$$

Let $F_{0}\left(i_{2}\right)$ to expression below,

$$
F_{0}\left(i_{2}\right)=n_{a v T}+2+i_{2}-1 \quad \text { for } 1 \leq i_{2} \leq n_{a v L} \quad \text { (8) Equation }
$$

(9) indicates the probability when the position of the first overflowed left-turn vehicle $O_{Q L J}(1)$ is equal to $j_{1}, j>1$.

$$
\begin{aligned}
& P_{O Q L J}\left(O_{Q L J}(1)=1\right)=C_{\left(F_{0}\left(n_{a L L}\right)\right)}^{n_{a L}} \rho_{L}^{n_{O L L}}\left(1-\rho_{L}\right)^{F_{0}\left(n_{a L L}\right)-n_{a L L}}
\end{aligned}
$$

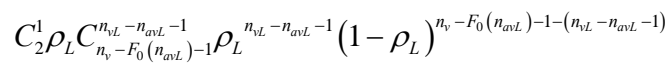

Equation (10) describes the probability of the first overflowed left-turn vehicle when $O_{Q L J}$ ( 1 ) is equal to $j_{1}$ and $j>1$.

$$
\begin{aligned}
& P_{O Q L J}\left(O_{Q L J}(1)=j_{1}\right)=C_{\left(F_{0}\left(n_{o L L}\right)\right)}^{n_{a_{L}}} \rho_{L}^{n_{\omega L L}}\left(1-\rho_{L}\right)^{F_{0}\left(n_{o L L}\right)-n_{O L L}}
\end{aligned}
$$

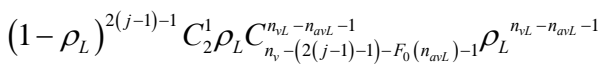

$$
\begin{aligned}
& \left(1-\rho_{L}\right)^{n_{v}-(2(j-1)-1)-F_{0}\left(n_{o L L}\right)-1-\left(n_{L L}-n_{o L L}-1\right)}
\end{aligned}
$$

According to the principle of conditional probability, when the flow and the rate of the different vehicle rate is given, the probability of the position of the first vehicle $O_{Q L J}$ (1) is equal to $j_{1}$ can be calculated by Equation (11).

$$
P_{i f}(A L \mid B L)=\frac{P_{i f}(A L \cdot B L)}{P_{i f}(B L)}=\frac{P_{O Q L}\left(O_{Q L J}(1)=j_{1}\right)}{P_{L}\left(n_{v L}\right)}
$$

As a result, the probability model of the queue position order of the left overflow first vehicle was obtained. Fig. 5 shows the example of the approach, seting parameters $n_{v}=50, n_{v L}=10, n_{a v}=5$, can be get the variation regular of $P_{i f}(A L \mid B L)$ with the the queue position order, as shown in Fig. 7.

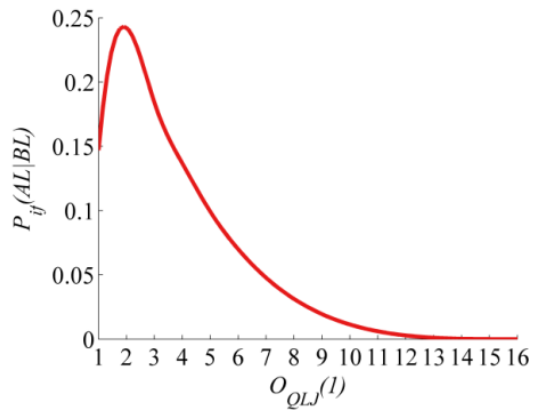

Fig.7 $P_{i f}(A L \mid B L)$ regularity of distribution, $n_{v}=50$

The probability of the queue position order of the left overflow first vehicle decreases as the increasing the 
queue position order, then the left overflow first vehicle more queues at the first location, also straight vehicle is possibly blocked. The expected number of blocked straight vehicle is the product accumulator by the number of blocked straight vehicle and the probability of the order queue position of the first left overflow vehicle, the expected number of blocked straight vehicle can express by equation (12).

$$
E\left(n_{v T_{-} J}\right)=\sum_{j_{1}=1}^{j_{1}=O_{Q L J_{-} \max }} P_{i f}(A L \mid B L) n_{v T}{ }^{\prime}\left(j_{1}\right)
$$

\section{Conclusion}

The design of stretching-segment have a significant effect on the efficience of vehicles'crossing at intersections in green phase. Based on the real traffic situation, the influence of the length of stretching-segment storage to straight-cross flow rate was explored in this study. The data showed that the duration and stability of saturation flow rate were highly influenced by the length of stretching-segment storage. A quantitative model which analyzed the effect of the length of stretching-segment to the flow rate and described the impact of vehicles in the upstream was proposed. Finally, the attenuate regulation of the straight-cross flow rate in green phase is developed by the analysis of vehivle's blocking.

\section{Acknowledgements}

This research was supported in part by the Programme of Introducing Talents of Discipline to Universities under grant B12022, National Nature Science Foundation of China under Grant 50878023, 51110305060, and the National High Technology Research and Development Program of China (No. 2011AA110304).

\section{References}

1. Clayton a J H, Road traffic calculations, J. Instn Civ. Engrs. 16 (1941) 247-248.

2. Webster F V, Traffic signal settings, road research technical paper No. 39. (Research Laboratory, Road, London,1958).

3. Akcelik R, Traffic signals: capacity and timing analysis. (ARRB, Australia, 1981).

4. Kimber R M, Semmens M C, Experiment to investigate saturation flows at traffic signal junctions, Traffic Engineering and Control, 23(3) (1982) 110-114, 117.

5. Robert M S, Using cumulative curves to measure saturation flow and lost time, Institute of Transportation Engineers, 58(10) (1988) 50-54.

6. TRB, Highway Capacity Manual 2000 (National Research Council, Washington DC, 2000).

7. Michalopoulos P G, O'connor J, Estimation of left-turn saturation flows, Transportation Research Record. (667) (1978) 35-41.

8. Soegondo T, Tumewu W, Kosasih D, Saturation flow, Fourth Conference of the Road Engineering Association of Asia and Australasia, Volume 5: Additional Papers and Conference Results. (Jakarta, Indones: Road Engineering Assoc of Asia \& Australasia, 1983) pp. 185-200.

9. Khosla K, Williams J C, Saturation flow at signalized intersections during longer green time, Journal of the Transportation Research Board. 1978 (2006) 61-67.

10. Wang M H, Benekohal R F, Analysis of left-turn saturation flow rates and capacity at signalized intersections, Transportation Research Record. (2173) (2010) 96-104.

11. Zhang W, Wang Y, Yang X, Influence of the number of through lane groups on saturation flow rates at signalized intersections, Proceedings of the Conference on Traffic and Transportation Studies, ICTTS. (Kunming, China: American Society of Civil Engineers, 2010),pp. 285-294.

12. Wuhong Wang, Yan Mao, Jin Jing, et al, Driver's various information process and multi-ruled decision-making mechanism: a fundamental of intelligent driving shaping model, International Journal of Computational Intelligence Systems, 4(3) (2011) 297-305.

13. Matson $\mathrm{T} \mathrm{M}$, the principles of traffic signal timing, trans. 18th ann. safety congr, 1929.

14. Matson T M, Smith W S, Hurd F W, traffic engineering. (mcgraw-hill book company Inc, New York,1955).

15. Hammond H F, Sorenson L J, traffic engineering handbook. (institute of traffic engineers and national Conservation Bureau, New York, 1941).

16. Evans H K, traffic engineering handbook (institute of traffic engineers and national Conservation Bureau, New Haven Connecticut, 1950).

17. Gazis D, Herman R, Maradudin A, the problem of the amber signal in traffic flow, Operation Research. 8 (1960) 112-132. 